\title{
LA PERTINENCE CONTEMPORAINE DE LA THÉORIE FICHTÉENNE DE LA RECONNAISSANCE
}

\author{
LUIS FELLIPE GARCIA
}

\begin{abstract}
This paper suggests the hypothesis that a new reading of Fichte's theory of recognition can enrich the contemporary debate on the subject, since it makes possible to link the question of recognition to the construction and maintenance of a community. We propose that this link would enlarge the juridicalpolitical field of the contemporary discussion so as to include in it a symbolic-cultural dimension of recognition.
\end{abstract}

\section{Introduction}

La reconnaissance (Anerkennung) est devenue depuis le début des années 1990 un opérateur conceptuel central de la pensée politique occidentale dont on remarque en effet l'influence sur plusieurs domaines de la réflexion, comme la sociologie, la philosophie, les sciences politiques, le droit, la psychologie et les sciences de la culture, entre autres. Sa portée a pris une telle ampleur qu'il n'est pas exagéré de dire que « la 'lutte pour la reconnaissance' est rapidement devenue la forme paradigmatique du conflit politique à la fin du XXème siècle $»^{1}$.

Le développement du concept de reconnaissance est historiquement lié à la justification de l'État de droit démocratique et renvoie à une tradition intellectuelle dont les grands maillons incluent Hobbes, Locke, Rousseau et Kant et qui abou-

1 Fraser Nancy, « Justice sociale, redistribution et reconnaissance », in Revue du MAUSS, n. 23, 2004, p. 152. 
tit à l'articulation d'une théorie systématique de la reconnaissance chez Fichte et Hegel..$^{2}$ Lattention portée à ce concept fut pourtant éclipsée à partir du milieu du XIXème siècle par l'avènement du marxisme et d'une pensée matérialiste, mettant en œuvre une puissante problématisation de la lutte des classes, qui est érigée à l'intérieur de cette tradition, en opérateur conceptuel fondamental de la pensée politique. Au cours des années 1930, la notion de reconnaissance refait ses premiers pas dans le scénario philosophique, à partir notamment des leçons hégéliennes d’Alexandre Kojève ${ }^{3}$ qui influencèrent toute une génération de penseurs français tels que Jacques Lacan, Jean Hyppolite, Georges Bataille, Maurice Merleau-Ponty et Jean-Paul Sartre ; son usage demeura pourtant limité, comme le fait remarquer Vladimir Safatle ${ }^{4}$, au niveau éthique et clinique.

Ce n'est qu'au début des années 1990, avec l'effondrement de l'Union Soviétique, que l'usage politique de ce concept a retrouvé sa potentialité ; en effet, le mouvement de démocratisation qui s’en est suivi a cherché à réarticuler la pensée politique autour d'un concept moins immédiatement rattaché aux déploiements du marxisme, comme létait celui de lutte des classes ; c'est dans ce contexte que la notion de reconnaissance sera logée au centre de la discussion politique à partir notamment des œuvres d'Axel Honneth et de Charles Taylor. ${ }^{5}$ Cette réappropriation politique de la reconnaissance, puissamment manifestée par la troisième génération de l'École de Francfort, s'effectue à travers un affaiblissement progressif de l'influence marxiste au profit, encore une fois, d'une relecture de Hegel souvent ponctuée d'un accent kantien. ${ }^{6}$ C'est également à partir de la pensée de Hegel que

2 Frischmann Bärbel, «Zum Begriff der Anerkennung - philosophische Grundlegung und pädagogische Relevanz ", in Sozialen Passagen, n. 1, 2009, p. 148.

3 Il s'agit d'une série de leçons sur la Phénoménologie de l'Esprit professées à l'École des Hautes Études de Sciences Sociales à Paris entre 1933 et 1939 et qui furent suivies par des personnalités telles que Jacques Lacan, Maurice Merleau-Ponty, Georges Bataille, Éric Weil, Raymond Aron, Jean Hyppolite, entre autres. Les lectures de Kojève reconstruisaient le système hégélien autour de la notion de reconnaissance.

4 Safatle Vladimir, O Circuito dos Afetos - Corpos políticos, desamparo e o fim do indivíduo, São Paulo, Cosac Naify, 2015, p. 286.

5 Même si Ludwig Siep en 1979 (Siep Ludwig, Anerkennung als Prinzip der praktischen Philosophie, Freiburg, Heder, 1979) et Andreas Wildt en 1982 (Wildt Andreas, Autonomie und Anerkennung. Hegels Moralitätskritik im Spiegel Fichterezeption, Stuttgart, Klett-Cota, 1982) exploraient déjà les enjeux politiques de la notion de reconnaissance, ce n'est qu'en 1992, avec les travaux d'Axel Honneth (notamment: Honneth Axel, Kampf um Anerkennung. Zur moralischen Grammatik sozialer Konflikte, Frankfurt, Suhrkamp, 1992) et de Charles Taylor (Taylor Charles, Multiculturalism: Examining the Politics of Recognition, Princeton, Princeton University Press, 1992) que la reconnaissance deviendra un concept majeur de la grammaire politique occidentale.

6 Laffaiblissement de l'influence marxiste au profit d'un retour à Kant et à Hegel est confirmé par la transformation des travaux de l'École de Francfort au fil des générations qui se succèdent : ainsi, si Marx était si puissamment présent dans les œuvres d'Adorno, d'Horkheimer et de Marcuse 
les limites de ce riche chantier conceptuel seront explorées en vue d'une refondation de la théorie de la reconnaissance afin de répondre aux critiques issues notamment de la tradition matérialiste. ${ }^{7}$

L'hypothèse avancée par cette étude suggère qu'une relecture de la théorie fichtéenne de la reconnaissance permettrait d'enrichir le débat contemporain autour de ce sujet dès lors qu'elle associe la question de la reconnaissance à celle de la construction d'un espace de partage. Nous suggérons l'idée qu'une telle approche permet d'élargir le terrain juridico-politique au sein duquel ce débat sarticule pour tenir compte d'une dimension symbolico-culturelle de la reconnaissance, en ouvrant l'horizon à la thématisation de blocages des processus de constitution identitaire. Le parcours argumentatif sera structuré de la façon suivante : (i) en partant des développements plus récents de la théorie de la reconnaissance apportés entre autres par la nouvelle génération de l'École de Francfort, ${ }^{8}$ on va en reconstruire les enjeux afin d'articuler les potentialités et une éventuelle limite de la perspective hégélienne ; ensuite, (ii) il sera question de revisiter, à partir de cet arrière-fond, la structure fichtéenne de la reconnaissance afin, finalement, (iii) d'explorer les possibles contributions d'une telle approche.

\section{L'articulation entre lutte et reconnaissance d'une perspective hégélienne}

Le début des années 1990, profondément marqué par la désintégration du bloc soviétique et par la subséquente accélération du processus de globalisation, fut aussi une époque de grand développement du droit international : les Droits de l'Homme ont fait un retour en force dans l'actualité, plusieurs facultés de re-

(première génération), on constate un mouvement de retour à Kant et aux questions de normativité chez Habermas (deuxième génération), auquel répondra une problématisation plus riche du contexte social au sein duquel opèrent les normes avancées par Honneth (troisième génération) à partir d'une relecture de Hegel. Laccent kantien de cette lecture hégélienne se manifeste dans la teneur normative de la conception de reconnaissance avancée par Honneth.

7 Cette perspective hégélienne peut être vérifiée même dans les tentatives les plus récentes de refondation d'une théorie de la reconnaissance : elle se manifeste par exemple chez Vladimir Safatle (Safatle V., "Towards an anti-predicative theory of recognition », in Grand Hotel Abyss - Desire, Recognition and the Restoration of the Subject, Leuven, Leuven University Press, 2016, pp. 271-299), et chez Robin Celikates (Bertram George W. et Celikates Robin, « Towards a Conflict Theory of Recognition: of the Constitution of Relations of Recognition in Conflict ", in European Journal of Philosophy, n. 23, 2015, pp. 838-861).

8 C'est-à-dire la génération qui succède à celle d'Axel Honneth : celle qui inclut aussi des auteurs comme Robin Celikates, Rahel Jaeggi, entre autres. 
lations internationales ont émergée et l'on a même cru, même si seulement de façon éphémère, en la capacité des Nations Unies à agir avec efficacité sur la scène internationale. ${ }^{9}$ Larticulation de la notion de « reconnaissance » comme opérateur conceptuel clé dans la rationalisation des demandes politiques a trouvé une résonnance précisément dans ce contexte de reprise de la foi en la normativité juridique et de déception devant les déploiements du marxisme : en effet, la reconnaissance, en tant qu'horizon de rationalisation de l'histoire, devient une sorte de remplaçant normatif du concept matérialiste de lutte des classes. ${ }^{10}$

Ce n'est donc pas un hasard si l'un des principaux axes critiques de la théorie de la reconnaissance vise justement à y réintroduire le concept de « lutte » et de « conflit ». ${ }^{11}$ Dans un article récent co-écrit avec Georg Bertram, Robin Celikates, l'un des principaux auteurs de la nouvelle génération de l'École de Francfort, identifie deux traditions majeures de la théorie de la reconnaissance : ${ }^{12}$ (i) l'une qu'il qualifie de positive, héritière de Fichte et de Hegel, qui aborde la reconnaissance dans sa dimension positive et émancipatrice et dont l'expression la plus aboutie est la théorie d'Axel Honneth ; (ii) et l'autre appelée, par contraste, négative, héritière de Rousseau et de Marx, au sein de laquelle la reconnaissance est critiquée en tant que structure idéologique empêchant une véritable émancipation, et dont l'expression contemporaine la plus aboutie est le travail de Judith Butler. D'un côté comme de l'autre, il existe, selon les auteurs, un défaut structurel empêchant de thématiser le conflit.

Chez Honneth, affirment les auteurs, il y a une insistance exagérée sur le normatif qui permet certes d'explorer la possibilité de lutte pour la reconnaissance (qui est bien le titre de son ouvrage célèbre), mais cette lutte se restreint à la quête de reconnaissance de certains attributs identitaires d'un sujet partiellement reconnu, c'est-à-dire le sujet $\mathrm{A}$ cherche à se faire reconnaître en tant que sujet ayant les attributs $\mathrm{x}, \mathrm{y}, \mathrm{z}$; cependant, dans la mesure où les rapports de reconnaissance

9 Cet éphémère instant de foi si rapidement dissout par l'éclatement de la Guerre du Golfe est bien saisi par le récit de Stanley Meisler - cf. Meisler Stanley, United Nations - A History, New York, Grove Press, 1995, pp. 157-177.

10 Pour une reconstruction du contexte social et intellectuel contribuant au remplacement de la notion de lutte des classes par celle de reconnaissance, voir le dernier chapitre de Grand Hotel Abyss, "Towards an anti-predicative theory of recognition ", cf. Safatle V., op. cit., pp. 271-299.

11 L'ouvre de Honneth, dans laquelle la notion de reconnaissance est pour la première fois articulée, s'intitule certes Lutte pour la reconnaissance; cependant, du fait que la constitution des sujets est essentiellement liée aux relations de reconnaissance, il doit toujours y avoir un degré minimum de reconnaissance pour que le sujet puisse entrer en conflit ; c'est pourquoi la théorie de Honneth s'avère insuffisante pour traiter la question de l'invisibilité sociale et les luttes propres aux sujets se trouvant dans cette situation - cf. Bertram G. et Celikates R., op. cit., p. 6. 
apparaissent comme une condition de possibilité de la constitution ontologique du sujet $\mathrm{A}$, une telle approche empêche de traiter la question d'un conflit pour la reconnaissance d'un sujet ontologiquement oblitéré, c'est-à-dire pour la reconnaissance d'un sujet en tant que sujet. ${ }^{13}$

Chez Butler, en revanche, il y a une puissante critique de la reconnaissance en tant que structure idéologique ayant des effets de marginalisation sociale politique ; cependant, on y trouve, d'après les auteurs, une exagération des effets politiquement pervers de la structure de la reconnaissance, qui rendent difficile l'exploration de l'horizon normatif à partir duquel l'on pourrait prendre un écart critique par rapport à la situation sociohistorique elle-même et engendrer des conflits. En d'autres termes, l'omniprésence de l'idéologie rend difficilement intelligible les impératifs à partir desquels on peut la critiquer et engendrer des conflits. ${ }^{14}$

Il y aurait ainsi, d'un côté, un défaut ontologique qui empêche la problématisation de la lutte de ceux qui sont ontologiquement oblitérés, de ceux qui ne sont pas reconnus en tant que sujets; de l'autre, un défaut normatif qui entrave l'exploration de l'horizon à partir duquel une prise de conscience critique par rapport aux idéologies serait possible. Face à ce défi, Celikates suggère une réinterprétation de la théorie hégélienne de la reconnaissance, qui serait susceptible de rétablir la centralité du conflit pour tout acte de reconnaissance, en signalant ainsi l'un des problèmes centraux de la réflexion philosophique contemporaine, à savoir : la nécessité de réarticuler le normatif et le socio-ontologique, l'idéalisme et le matérialisme, la reconnaissance et la lutte des classes.

Afin de réarticuler la normativité contenue dans la notion de reconnaissance avec la problématisation des structures idéologiques entravant son accomplissement, il faut que le schème de la reconnaissance puisse thématiser les échecs issus de la mise en œuvre de la structure même qui la rend possible; en outre, il est

13 Même si Honneth problématise la question de l'invisibilité sociale, ses analyses, portant presque exclusivement sur les mécanismes épistémologiques empêchant la vision, ne thématisent guère la lutte de ceux qui sont relégués à cette invisibilité ; autrement dit, la lutte pour la reconnaissance d'un sujet ontologiquement oblitéré n’est pas traitée - voir : Honneth A., «Invisibilité : sur l'épistémologie de la "reconnaissance" ", in Revue du Mauss, n. 23, 2004, pp. 137-151. D’après Bertram et Celikates eux-mêmes: « We can summarize the tensions in Honneth's approach as follows: Honneth does not manage to unify the social-ontological and normative-critical aspects of the concept of recognition. To the extent that recognition is established as a normative-critical concept, it becomes unclear how it functions as a basic concept with social-ontological import. Doesn't Honneth have to deal with the possibility that practices can be utterly forgetful of recognition or that individuals remain utterly socially invisible? How can subjects in such a situation enter into conflicts, insofar as their constitution depends on relations of recognition? » (cf. Bertram G. et Celikates R., op. cit., p. 844).

14 Selon Bertram et Celikates: « it becomes unintelligible, under conditions of comprehensive normalization, where the resources come from that enable agents to enter into conflicts at all - i.e., that ground their capacity for conflict in general. ». (Ibid., p. 845) 
nécessaire que le schème de la reconnaissance renferme la possibilité d'être abordé aussi bien au niveau de la normativité qu'il met en marche qu'à celui des effets socio-ontologiques (l'oblitération de sujets) qu'il comporte, puisqu'autrement on ne saurait problématiser les échecs issus de la propre mise en marche du processus de reconnaissance. C’est sur ce point que le système hégélien manifeste sa fertilité.

Une reprise de la première thèse du chapitre central de la Phénoménologie de l'Esprit concernant la reconnaissance, celui portant sur la relation maîtrise-servitude, nous permet de visualiser les échecs issus de la structure de la reconnaissance. Ce célèbre chapitre commence ainsi: «La conscience de soi nest en soi et pour soi quand et parce quelle est en soi et pour soi pour une autre conscience de soi ; c'est-à-dire quelle n'est qu'en tant quêetre reconnu [als ein Anerkanntes] »15.

Hegel avance l'idée que la conscience au sens strict ne surgit que dans un contexte intersubjectif fondamentalement réciproque, c'est-à-dire que la conscience doit reconnaître qu'elle est reconnue par une conscience qu'elle reconnaît. Autrement dit, tant que l'intersubjectivité n’aboutit pas à une telle réciprocité, ce qui résulte d'un tel rapport ne saurait être appelé au sens strict « conscience». Or, si ceux qui sont engagés dans une relation intersubjective non-réciproque ne se constituent pas à proprement parler comme consciences, que sont-ils alors?

Voici la réponse qui ressort du texte hégélien :

L'individu qui n'a pas mis sa vie en jeu peut bien être reconnu comme personne; mais il n’a pas atteint la vérité de cette reconnaissance comme reconnaissance d'une conscience indépendante [als eines selbständigen Selbstbewusstseins $]^{16}$.

Autrement dit, un individu peut être reconnu comme personne sans pour autant se faire reconnaitre comme conscience de soi ; cela veut dire que la structure de la reconnaissance, même lorsqu'elle habilite l'accueil d'autrui en tant quêtre humain, peut néanmoins rester bloquée à ce niveau et ne pas parvenir au niveau de la saisie d'autrui en tant que conscience indépendante aussi longtemps qu'une condition supplémentaire ne sera pas remplie, à savoir : la mise en jeu de sa propre vie. En l'absence d'une telle condition, la structure de la reconnaissance, en tant

15 Hegel Georg Wilhelm Friedrich, Phénoménologie de l'Esprit, trad. par J. Hyppolite, Paris, Aubier, 1941, t. I, p. 155. Texte original: «Das Selbstbewusstsein ist an und für sich, indem und dadurch, dass es für ein Anders an und für sich ist; d. h. es ist nur als ein Anerkanntes » (Hegel G. W. F., Phänomenologie des Geistes - Werke 3, Suhrkamp, 1986, p. 145).

16 Hegel G. W. F., Phénoménologie de l’Esprit, op. cit., t. I, p. 159. Texte original : « Das Individuum, welches das Leben nicht gewagt hat, kann wohl als Person anerkannt werden; aber es hat die Wahrheit dieses Anerkanntseins als eines selbständigen Selbstbewusstseins nicht erreicht » (Hegel G. W. F., op. cit., p. 149). 
que mise en œuvre d'un rapport symétrique entre consciences, est vouée à l'échec dès lors que l'indépendance ou l'autonomie (Selbständigkeit) de la conscience demeurera non-reconnue.

Cette dimension de la pensée hégélienne s'avère fondamentale pour l'articulation des notions de reconnaissance et de lutte, puisqu'en effet, en l'absence d'une véritable lutte pour la liberté (une lutte dans laquelle on est prêt à risquer sa vie), la reconnaissance réciproque des consciences autonomes ne saura advenir; en d'autres termes, la lutte se révèle un composant constitutif de la réciprocité de la reconnaissance. Il y a dès lors une interdépendance entre l'opérativité de la structure normative (reconnaissance) et l'engagement social effectif (lutte), ce qui indique les potentialités de la théorie hégélienne pour combler le double défaut, normatif et socio-ontologique, issu de la discussion contemporaine autour de la reconnaissance.

C’est précisément à cet engrenage de la machinerie hégélienne que puiseront des auteurs comme Robin Celikates, Vladimir Safatle et, bien avant eux, Frantz Fanon, pour repenser le schéma de la reconnaissance en y insérant des questions urgentes de la contemporanéité. En effet, ce rapport constitutif entre reconnaissance et capacité de conflit (Konfliktfähigkeit) chez Hegel servira de base à Celikates pour articuler la nécessité d'une flexibilisation des institutions afin d'y intégrer la possibilité de remise en cause des normes de reconnaissance; ${ }^{17}$ c'est également du rôle clé joué, au sein du système hégélien, par le courage de risquer sa vie dans le processus de constitution identitaire que s'inspirera Safatle pour souligner l'existence d'une dimension affective (pré-prédicative) antérieure à toute identité constituée, ce qui ouvre l'horizon politico-épistémologique de la reconnaissance à des modèles de subjectivation irréductibles à l'individualisme ;18 de même, cette idée hégélienne selon laquelle la lutte à mort pour la liberté est une condition nécessaire du passage de la reconnaissance comme personne à la reconnaissance comme conscience a servi à Frantz Fanon pour dénoncer la persistance du rapport maître-esclave au sein de la structure psychique de la relation entre Noirs et Blancs - au sein de laquelle les Noirs sont reconnus comme personnes, mais pas encore comme consciences. ${ }^{19}$

Bertram G. et Celikates R., op. cit., p. 864.

18 Safatle V., Grande Hotel Abismo, op. cit., p. 322.

19 Frantz Fanon, dans son puissant étude des effets psycho-sociaux de la colonisation, trouve un exemple historique de l'essentialité du rapport entre lutte et reconnaissance réciproque de consciences autonomes ; il affirme ainsi qu' « historiquement, le nègre plongé dans l'inessentialité de la servitude, a été libéré par le maître. Il n’a pas soutenu la lutte pour la liberté. [...] Le nègre est un esclave à qui on a permis d'adopter une attitude de maître. Le Blanc est le maître qui a permis à ses esclaves de manger à sa table. »-cf. Fanon Frantz, Peau noire, masques blancs, Paris, Editions du Seuil, 1952, p. 212. Autrement dit, tant que la libération de l'esclave ne constitue pas le résultat 
On voit que cette articulation apportée par le système hégélien entre l'avènement de la conscience et le courage de risquer sa vie dispose d'une puissance conceptuelle dans laquelle des branches diverses de la pensée contemporaine (comme la théorie critique, la psychologie sociale et le post-colonialisme) peuvent puiser pour repenser, voire refonder, la théorie de la reconnaissance en y articulant, d'un côté, la dimension proprement normative de l'accueil d'autrui au sein d'un système de normes et, de l'autre, la dimension socio-ontologique du conflit pouvant éventuellement remettre en cause le système normatiflui-même. D’une part, la dimension normative de la reconnaissance n'empêche pas la thématisation des luttes de subjectivités oblitérées, dès lors que la lutte est précisément ce qui rend possible la reconnaissance des consciences (et donc la reconnaissances des sujets en tant que sujets); d'autre part, la dimension socio-ontologique n'empêche pas la prise de conscience critique, puisque, bien qu'il soit pris dans le monde du seigneur, le serf peut toujours engendrer un conflit avec ce monde, soit directement à travers l'affrontement de la mort, soit indirectement, comme indique le célèbre chapitre de Hegel, à travers le travail.

Si l'arrière-fond hégélien est certes puissant pour remettre à l'ordre du jour la question de la lutte, il semble pourtant moins performant lorsqu'il est question de cerner les défauts structurels bloquant le rapport à soi et délaborer une stratégie d'action pour les combattre ; en effet, selon une image célèbre avancée par Hegel dans la Phénoménologie de l'Esprit:

Les blessures de l'esprit [die Wunden des Geistes] se guérissent sans laisser des cicatrices. Le fait n'est pas l'Impérissable, mais l'esprit le réabsorbe en soi-même [von dem Geiste in sich zurückgenommen], et le côté de la singularité qui, soit comme intention, soit comme sa négation et sa borne dans lélément de lêtre-là, est présent dans le fait, est ce qui immédiatement disparaît ${ }^{20}$.

Autrement dit, l'Esprit constitue ce qui dissout les singularités des faits historiques en permettant de les re-signifier de la perspective de la totalité du mouvement; de cette perspective du tout, les blessures (les particularités bloquant le processus) sont réabsorbées par l'Esprit et incorporées dans son mouvement. Cela

d'une véritable lutte, la structure de la reconnaissance demeure asymétrique et l'esclave reste esclave puisque son indépendance et son autonomie ne sont pas véritablement reconnues.

20 Hegel G. W. F., Phénoménologie de l’Esprit, op. cit., t. II, p. 197. Texte original : « Die Wunden des Geistes heilen, ohne dass Narben bleiben; die Tat ist nicht das Unvergängliche, sondern wird von dem Geiste in sich zurückgenommen, und die Seite der Einzelheit, die an ihr, es sei als Absicht oder als daseiende Negativität und Schranke derselben vorhanden ist, ist das unmittelbar Verschwindende. » (Hegel G. W. F., op. cit., p. 492). 
veut dire que sur la longue durée, l'Esprit, grâce à ce pouvoir de réabsorption dépassant les singularités, tend au dépassement et même à la réintégration des obstacles qui les bloquent. Cette tendance de l'Esprit à s'affranchir de ses entraves se manifeste dans des outils conceptuels clefs du système hégélien, tels que la notion de " ruse de la raison ${ }^{21}$, développée dans sa philosophie de l'histoire, ou l'omniprésent opérateur conceptuel de l'Aufhebung. ${ }^{22}$

La tradition matérialiste, fort influencée par l'hégélianisme, critiquera précisément ce point du système. Déjà le jeune Marx dans sa critique de la philosophie politique de Hegel insista sur le fait que l'État, l'institution devant réaliser l'Aufhebung des contradictions de la société civile, au lieu de dépasser les entraves incarnées dans les conflits sociaux, tend plutôt, en fonction des fractures socioéconomiques à l'intérieur de la société civile, à reproduire les contradictions à un autre niveau ; puisqu'en effet, il s’avère que la domination exercée par une classe (les propriétaires) au niveau de la société civile tend à se traduire par une domination au niveau de l'État où les propriétaires contrôleront le pouvoir politique, de sorte que, d'après Marx, l'État peut prendre la forme d'une propriété privée au sein de laquelle les contradictions de la société civile, au lieu dêtre dépassées, tendent plutôt à se consolider. ${ }^{23}$ En d'autres termes, la guérison naturelle et sans cicatrices de l'Esprit se révèle dans ce cas plutôt comme une reproduction de la blessure.

21 Dans les manuscrits de ses cours de philosophie de l'histoire, Hegel explique que la ruse de la Raison consiste dans le fait que même si « c'est leur bien propre que peuples et individus cherchent et obtiennent dans leur agissante vitalité, [...] ils sont les moyens et les instruments d'une chose plus élevée, plus vaste qu'ils ignorent et accomplissent inconsciemment » (cf. Hegel G. W. F., La Raison dans l'Histoire, trad. par K. Papaioannou, Paris, Plon, 1965, p. 110) ; cet accomplissement inconscient de ce qui est parfois nommé « Esprit du Monde » ou "Raison immanente dans l'Histoire » constitue précisément « la ruse de la Raison », ce qui, de la perspective de l'Absolu, peut être nommé la « ruse absolue » ou même « la Providence divine » (cf. Hegel G. W. F., Encyclopédie des sciences philosophiques I - La science de la logique, trad. par B. Bourgeois, Paris, Vrin, 2014, p. 614). Cette notion, en ceci qu'elle implique une sorte d'inconscient collectif (bewusstlose Instikte der Menschen), contient certes un puissant outil épistémologique pour reconstruire l'histoire ; cependant elle manifeste également une considérable confiance dans la tendance de l'Esprit à dépasser les obstacles à son accomplissement progressif.

22 Selon Hegel, «Par aufheben nous entendons d'abord la même chose que par hinwegräumen (abroger), negieren (nier). [...] Mais, en outre, aufheben signifie aussi la même chose que aufbewahren (conserver)» - cf. Hegel G. W. F., Encyclopédie des sciences philosophiques I, op. cit., p. 530 ; c'est ce double mouvement de négation et conservation qui permet à l'Esprit de dépasser les obstacles tout en les incluant dans son mouvement.

23 Comme le formule Marx lui-même dans sa Critique de la philosophie politique de Hegel : « Le fait que la propriété privée se rattache à la fonction publique se traduit dans le majorat en ce que l'existence de l'Etat semble être une inhérence de la propriété privée immédiate, de la propriété foncière, un accident de celle-ci. », cf. Marx Karl, Euvres, Paris, Gallimard, Bibliothèque de la Pléiade, t. III, p. 997. Autrement dit, l'Etat ne serait plus la concrétisation d'une structure au sein de laquelle « mon intérêt particulier et mon intérêt substantiel sont conservés et maintenus dans le but d'un autre » 
De même, la structure de la critique marxienne est similaire en ce qui concerne le «travail », du fait qu'il devrait, d’après le système hégélien, entamer un mouvement de dépassement (Aufhebung) de l'asymétrie du rapport entre maître et esclave. En effet, selon le jeune Marx, le travail - qui idéalement est cette opération décrite par Hegel permettant à la conscience « de se redécouvrir elle-même par elle-même $»^{24}$ - devient souvent, au sein des rapports économiques asymétriques, l'activité par laquelle le travailleur est rendu « esclave de son objet » ${ }^{25}$ et du " maitre de son travail ${ }^{26}$, dans un processus que Marx appelle l'aliénation du travail ;27 autrement dit, lopération qui devrait mettre en marche le mouvement de dépassement d'asymétrie entre les consciences peut, à l'inverse, en creuser le fossé. C’est pourquoi l'Aufhebung, censée dépasser la contradiction, ne fait encore une fois que la reproduire à un niveau où elle est plutôt élargie. ${ }^{28}$

On peut ici reconnaître le même schéma critique que celui auquel feront appel les théories " négatives » de la reconnaissance, c'est-à-dire celles qui soutiennent que l'idée de reconnaissance introduit une structure empêchant la réalisation de la liberté. Il s'agit en effet de montrer comment l'activité censée dépasser (dans le sens d'Aufhebung) une asymétrie intersubjective finit par la reproduire de façon systématique. C'est pourquoi la critique de la notion de reconnaissance puise constamment dans les outils conceptuels propres à la tradition matérialiste lorsqu'il est question de problématiser ces blessures qui, au lieu de "guérir sans laisser de cicatrices ", finissent par se multiplier. C’est précisément dans le but de thématiser ces blessures systématiques - que j’appellerai ici, faute de mieux, « des blocages » - et d'ouvrir ainsi le terrain à lélaboration de stratégies pratiques pour

(cf. Hegel G. W. F., Principes de la philosophie du droit, trad. par A. Kaan, Paris, Gallimard, 1940, p. 261) en permettant ainsi le dépassement des contradictions d'intérêts particuliers exprimées dans la société civile, mais plutôt un mécanisme de reproduction de ces mêmes contradictions.

l'homme " étranger à son propre corps, au monde extérieur aussi bien qu'à son essence spirituelle, à son essence humaine ", cf. Marx K., Manuscrits de 1844, op. cit., p. 116.

28 du dépassement » issue de Hegel avec la notion de survivance du dépassé (aufgehoben), où il est justement question des structures positives et actives conditionnant nos rapports à nous-mêmes et nos propres passés, cf. Althusser Louis, Pour Marx, Paris, Maspero, 1965, pp. 114-116. Adorno s'attaque également à la logique de l'Aufhebung qui incarne la tendance à dépasser le conflit entre l'identique et le non-identique sous la figure de l'identique, ce qui implique un mépris du particulier et de la différence, qu'Adorno prétend remettre en valeur avec sa dialectique dans laquelle le négatif n'est pas dépassé par l'Aufhebung, cf. Adorno Theodor W., Dialectique négative, trad. par G. Coffin, J. Masson, O. Masson, A. Renaut et D. Trousson, Paris, Payot, 1978. 
les affronter que l'on va faire jouer le cadre conceptuel fichtéen qui, d'après notre hypothèse, pourrait apporter d'importants outils pour repenser l'articulation entre le socio-ontologique et le normatif sans pour autant tomber dans le piège d'un mouvement d'auto-guérison de l'esprit - une articulation entre les blocages matérialistes et l'idéalisme de la conscience.

\section{L'approche fichtéenne}

Au début des années 1990, Daniel Breazeale, l'un des responsables du développement récent de la Forschung fichtéenne dans le monde anglo-saxon, écrivit un court mais influent article intitulé "Why Fichte now ? ${ }^{29}$, dans lequel il énumérât les raisons pour lesquelles les études fichtéennes bénéficiaient à l'époque d'une remarquable renaissance. ${ }^{30}$ Breazeale souligne, parmi d'autres raisons, (i) la priorité du pratique sur le théorique, (ii) la thèse de l'intersubjectivité comme une condition de possibilité de la conscience et (iii) les limites souvent dénoncées par Fichte

29 Breazeale Daniel, «Why Fichte now? », in The Journal of Philosophy, v. 88, n. 10, 1991, pp. 524-531.

30 Au cours de ces dernières années, cette renaissance a connu une impulsion renouvelée dans diverses régions du globe ; aux Etats-Unis, Tom Rockmore trouve chez Fichte des outils conceptuels pour construire une philosophie de l'activité comme alternative à la philosophie hégélienne du concept (Fichte, Marx and the German Philosophical Tradition) ; en France, Jean-Christophe Goddard y souligne le cadre conceptuel psychanalytique qui ouvre la porte à des problématisations sur la contingence et les blocages dans les mouvements de l'esprit (La philosophie fichtéenne de la vie, le transcendantal et le pathologique) ; en Belgique, Marc Maesschalck met l'accent sur la dimension émancipatrice du projet fichtéen (Droit et création sociale chez Fichte), Quentin Landenne y propose l'articulation d'un suggestif perspectivisme transcendantal, capable de penser une politique perspectiviste qui ne perde pas de vue son horizon transcendantal (Le perspectivisme transcendantal de Fichte), tandis qu'Augustin Dumont explore pour sa part l'originalité du paysage conceptuel inauguré par la philosophie fichtéenne et déployé par le romantisme allemand en ce qui concerne notamment les rapports entre imagination et langage (Lopacité du sensible chez Fichte et Novalis : Théories et pratiques de l'imagination transcendantale à lépreuve du langage) ; en Italie, Alessandro Bertinetto explore les instruments conceptuels pour penser une philosophie de l'imagination, en guise d'alternative à une philosophie de la raison, à partir notamment des dernières versions de la WL (La forza dell'immagine : argomentazione transcendentale e ricorsività nelle filosofia di J.G. Fichte) ; en Allemagne, la redécouverte de ses exposés de philosophie populaire a motivé l'exploration de la dimension pédagogique du système fichtéen, bien incarnée dans les travaux de Peter Österreich et Harmut Traub (J.G. Fichtes Populärphilosophie et plus récemment Der ganze Fichte : die populäre, wissenschaftliche und metaphilosophische Erschließung der Welt); au Japon, la création récente de Japanische Fichte-Gesellschaft suit le chemin ouvert par Nishida Kitaro, fondateur de l'école de Kyoto, qui a proposé une originale interprétation de la Tathandlung où il trouvait des éléments proches de la philosophie bouddhiste; en Amérique Latine, la récente création, en 2010, de l'Asociación Latinoamericana de Estudios sobre Fichte (ALEF) a multiplié le nombre de chercheurs se consacrant aux études fichtéennes et tend à ouvrir des portes pour une approche renouvelée de la pensée du philosophe. 
de la raison spéculative. C'est aussi sur cette insistance sur l'intersubjectivité où le pratique prend la relève sur la raison théorique (au sens kantien) et même sur la spéculation (au sens hégélien), que s’appuya Tom Rockmore pour traiter des univers conceptuels de Fichte et de Marx comme des sources permettant d'articuler une philosophie de l'activité par opposition à une philosophie du concept de type hégélienne. En effet, comme le souligne cet auteur, les questions philosophiques fondamentales, aussi bien chez Marx (l'autoréalisation de l'homme) que chez Fichte (l'identité métaphysique entre le moi individuel et la conscience absolue ${ }^{31}$ ), ne pourront être résolues que par la mise en cuvre d'une pratique collective. ${ }^{32}$ En s'appuyant sur ces points soulevés par Breazeale et Rockmore, on prétend avancer que la théorie fichtéenne de l'intersubjectivité peut, en fonction de sa façon particulière d'articuler idéalisme et matérialisme, ${ }^{33}$ enrichir les discussions contemporaines portant sur la reconnaissance, particulièrement en ceci qu'elle nous permet d'envisager lélaboration de stratégies collectives de lutte face aux défauts structurels des schèmes de reconnaissance.

Nous voulons suggérer qu'une nouvelle perspective peut s'ouvrir autour des discussions contemporaines concernant la reconnaissance, en fonction notamment de la distinction fichtéenne entre deux moments de la reconnaissance : (i) la position du problème issu de la seule présence d'autrui et (ii) le moment pragmatique de la mise en cuvre d'une praxis pour affronter le problème. ${ }^{34}$ Suivant cette hypothèse, une telle distinction permet de réarticuler le normatif avec le socio-ontologique grâce au rapprochement de la question de la reconnaissance de celle de la constitution d'une communauté, ce qui permet d'affronter les problèmes des

31 L'«identité métaphysique », selon l'auteur, ne se réduit ni à l'identité numérique, l'identité d'une chose à elle-même, ni à l'identité qualitative, celle entre deux choses à légard d'une propriété commune ; mais elle doit être comprise plutôt comme « a nonnumerical, nonqualitative unity in difference, which is brought about by the subject between the subject and the object it generates through its activity " (cf. Rockmore Tom, Fichte, Marx and the German Philosophical Tradition, Southern Illinois University Press, 1980, p. 69).

32 Ibid., pp. 84-86.

33 En effet, comme le fait remarquer Jean-Marie Vaysse, « l'objectif de la Wissenschaftslehre est de renvoyer dos à dos l'idéalisme kantien et le dogmatisme spinoziste " (cf. Vaysse Jean-Marie., "Dynamique et subjectivité selon Fichte : effort, pulsion, aspiration » in Revue Germanique, n. 18, 2002, p. 158), il s'agit d'articuler une tradition d'après laquelle le sujet est constituant (l'idéalisme) avec une autre selon laquelle le sujet est plutôt constitué, c'est-à-dire qu'il est secondaire par rapport au principe de sa constitution (ce que l'on a appelé ici " tradition matérialiste »); sur ce projet de synthèse entre Kant et Spinoza, voir aussi l'étude récemment parue d'Allen Wood : Wood Allen, Fichte's Ethical Thought, Oxford, Oxford University Press, 2016, pp. 5-6.

34 Concernant la centralité du problème de l'intersubjectivité dans la pensée fichtéenne à l'époque de Iéna, voir en particulier les excellentes études d'Alexis Philonenko (Philonenko Alexis, La liberté humaine dans la philosophie de Fichte, Paris, Vrin, 1966) et Reinhard Lauth (Lauth Reinhard, « Le problème de l'interpersonnalité chez Fichte ", in Archives de Philosophie, v. 25, n. 3/4, pp. 325-344, 1962). 
blocages systématiques grâce à la problématisation du rôle central de la production conceptuelle au sein du processus de constitution de chaque communauté.

Voyons d'abord en quoi consistent ces deux moments identifiés par Fichte pour pouvoir, dans la session suivante, en extraire les potentielles contributions à la discussion contemporaine. Premièrement, selon le penseur de Rammenau, la genèse de la conscience de soi dépend du fait que lêtre humain trouve une espèce particulière de résistance au déploiement de sa puissance ; résistance qui ne saurait être posée que par la seule présence d'un autre être libre. Fichte l'explique dans la Grundlage des Naturrechts nach Principien der Wissenschaftslehre de la façon suivante :

[A] u fondement de toute action réciproque arbitraire dêtres libres, il y a de leur part une action réciproque originaire et nécessaire, à savoir celle-ci : lêtre libre force [nötigt] par sa simple présence dans le monde sensible, sans plus [durch seine blosse Gegenwart in der Sinnenwelt, ohne weiteres], chaque autre être libre à le reconnaître pour une personne. Il donne le phénomène déterminé, l’autre donne le concept déterminé. L'un et l'autre sont réunis nécessairement, et la liberté n’a pas dans cette affaire le moindre rôle à jouer. Il en naît une connaissance qu'ils ont en commun, et rien de plus. Tous deux se reconnaissent réciproquement en leur intériorité, mais ils sont isolés, comme auparavant ${ }^{35}$.

Il en ressort que la simple présence corporelle d'autrui dans l'horizon du déploiement de la puissance humaine d'action pose immédiatement un problème, à savoir celui de la résistance au déploiement de la puissance. Le ressenti de cette

35 Fichte Johann Gottlieb, Fondement du droit naturel selon les principes de la doctrine de la science, trad. par A. Renaut, Paris, PUF, 1984, pp. 100-101. Texte original : " Aller willkürlichen Wechselwirkung freier Wesen, liegt eine ursprüngliche und nothwendige Wechselwirkung derselben zum Grunde, diese: das freie Wesen nöthigt durch seine blosse Gegenwart in der Sinnenwelt, ohne weiteres, jedes andere freie Wesen es für eine Person anzuerkennen. Es giebt die bestimmte Erscheinung, der Andere giebt den bestimmten Begriff. Beides ist nothwendig vereinigt, und die Freiheit hat dabei nicht den geringsten Spielraum. - Hierdurch entsteht eine gemeinschaftliche Erkenntniss, und nichts weiter. Beide erkennen einander in ihrem Inneren, aber sie sind isoliert, wie zuvor. » (Fichte J. G., Gesamtausgabe der Bayerischen Akademie der Wissenschaften, éd. par Erich Fuchs, Hans Gliwitzky, Reinhard Lauth et Peter K. Schneider, Reihe I : Werke II, Reihe II : Nachgelassene Schriften Reihe III : Briefe, Reihe IV : Kollegnachschriften, Stuttgart, Frommann-Holzboog, 19622012, Reihe I, t. 3, p. 384 - dorénavant: GA). Ce passage se trouve au $\$ 7$ de l’ouvrage qui conclut la déduction de l'applicabilité du concept de droit. Il s'ensuit dès lors (i) de la déduction du concept de droit $(\$ \$ 1-4)$, basée sur le fait que l’attribution à soi-même d’une causalité libre conduit à la question de la limitation réciproque de libertés constituant ce que Fichte appelle une "relation juridique »; et (ii) des deux premières conditions de son applicabilité, à savoir l'attribution à soi-même d'" un corps matériel articulé [materiellen Leib] » (\$5) et la position de son propre corps sous l'influence d’autrui (\$6), que la relation juridique s’inscrit nécessairement dans le monde matériel. Ce parcours conduit de la sorte à la question de savoir « comment une communauté dêtres libres en tant que tels est possible?» (\$7). 
résistance issue de la présence d'autrui est immédiat, de sorte que "la liberté n’a pas dans cette affaire le moindre rôle à jouer ", la résistance est là par la seule présence de l'autre, sans plus (ohne weiteres), c'est pourquoi elle constitue en quelque sorte une " action réciproque originaire".

De plus, de cette " action originaire ", il découle une connaissance en commun, ou un premier moment de reconnaissance, où les sujets restent pourtant isolés. Autrement dit, on y trouve une résistance réciproque, mais cela ne se traduit pas encore dans la constitution d'un espace intersubjectif. En effet, si la simple présence d'autrui conduisait immédiatement à la concrétisation de l'autolimitation réciproque, alors aucune conscience de la liberté ne saurait y advenir, mais seulement celle d'un instinct agissant de façon nécessaire face aux résistances trouvées ; dès lors, la conscience ne ressort de ce rapport intersubjectif que si la limitation nest pas prédéterminée, mais tout simplement exigée par la présence sensible de ce qui résiste à la force - une telle présence constitue un " appel [Aufforderung] à agir adressé au sujet » ${ }^{36}$. Par conséquent, ce premier moment articule seulement une sorte de saisie de la limitation originaire, et pas encore un concept du modus operandi de la limitation - c'est-à-dire aucune limite effective n’est encore donnée et ils demeurent «isolés, comme auparavant ».

Lêtre humain est ainsi toujours déjà inscrit dans l'entrecroisement d'appels mutuels issus de la présence d’autres êtres humains et le premier moment de la reconnaissance se joue au niveau du surgissement $d u$ simple concept d'autres sujets, bien que la façon dont ce concept détermine mes actions ne soit pas encore établie. Il en ressort que la saisie de l'appel d'autrui renferme deux aspects constitutivement liés : (a) celui de la saisie des limites de ma force et donc de ma propre finitude et (b) celui de la saisie de la similarité entre ma force et celle qui me résiste. Ainsi, une fois que le moi et son autre (le toi) révèlent l'un à l'autre leurs finitudes et leurs similarités, l’appel issu de la présence d'autrui conduit à la reconnaissance du problème de la construction d'un espace de coexistence. Ce premier moment de la reconnaissance peut être ainsi illustré :

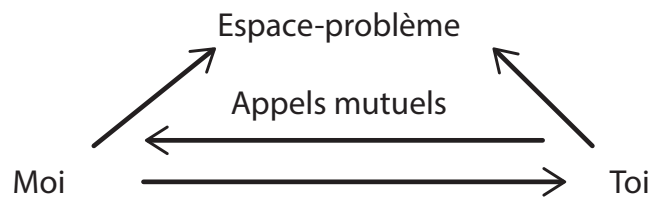

36 Fichte J. G., Fondement du droit naturel selon les principes de la doctrine de la science, op. cit., p. 49 (GA, I, 3, p. 342). 
Le résultat du premier moment indique ainsi que la reconnaissance ne saurait s'achever qu'à travers l'engagement réciproque dans la construction d'un espace d'intersubjectivité, espace où le moi et le toi ne seront plus « isolés, comme auparavant».

Le deuxième moment de la reconnaissance est celui qui pose le problème proprement politique. Si, comme on l'a vu au sein du premier moment, la seule présence d'autrui implique une connaissance en commun sans engagement mutuel, alors l'établissement relation d'autolimitation réciproque effective de libertés ne saurait se réaliser à moins "qu'une communauté entre des êtres libres comme tels [...puisse] trouver une existence durable $»^{37}$, puisque la reconnaissance réciproque ne s'opère de façon durable que « sous la condition et pour le cas où une communauté, une activité d'influence réciproque, doit exister entre des êtres libres comme tels, sans dommage pour leur liberté ${ }^{38}$. Cela veut dire qu'une reconnaissance réciproque durable exige que les êtres libres, auparavant isolés, entrent en communauté, car ce n'est que celle-ci qui peut rendre effective la reconnaissance mutuelle.

Dès lors, si le premier moment de la reconnaissance porte sur le surgissement d'un concept de soi-même et d'autrui opérant ainsi sur le niveau du savoir mutuel, le second moment porte sur l'engagement réciproque et opère sur le niveau du vouloir mutuel : d'un côté, il est question de la constitution nécessairement intersubjective du savoir de soi (le premier moment de la reconnaissance, celui de l'appel), de l'autre, de l'engagement effectif dans la construction volontaire d'un espace de partage (le moment de concrétisation de la reconnaissance, celui de la construction de la communauté) ${ }^{39}$. Il est important de souligner que le premier moment est la condition du second, c'est-à-dire que ce n'est qu'en fonction de l'appel issu de la présence d'autrui que le problème de la construction se pose, tandis que le second moment est celui où la reconnaissance peut effectivement s'accomplir,

\footnotetext{
Ibid., p. 103 (GA, I, 3, p. 385).

Ibid., p. 106 (GA, I, 3, p. 388).

39 L'importance d'un «engagement mutuel de volontés » au sein d'un processus commun de réalisation de la communauté caractéristique de ce deuxième moment de la reconnaissance est mise en avant par Marc Maesschalck dans son excellente reconstruction de la structure fichtéenne de la reconnaissance. Il s'agit de montrer que l'articulation de deux moments va au-delà de tout schématisme de type kantien puisque, dans la mesure où elle implique l'investissement affectif mutuel, son opérativité est constitutivement liée aux enjeux de chaque contexte ; ce que Fichte avance serait, ainsi, non un schématisme de la reconnaissance, mais plutôt une typique de la reconnaissance - c'est pourquoi comme l'affirme l'auteur, «le concept de l'autre est insuffisant pour expliquer la communauté dêtres libres, il faut encore un engagement concret pour la reconnaissance d'autrui ", un schéma conceptuel de la reconnaissance d'autrui ne s'avère pas suffisant, il faut qu'il existe une typique opérant sur les vouloirs réciproques; $\mathrm{cf}$. Maesschalck Marc, Normes et Contextes - les fondements d'une pragmatique contextuelle, Hildesheim / Zürich / New York, Georg Olms Verlag, 2001, pp. 205-231.
} 
puisque ce n'est que dans la construction de l'espace commun que le problème posé par le premier moment peut trouver une solution effective.

Si la Grundlage des Naturrechts (GNR), en vertu de la portée de l'ouvrage, va explorer la condition juridique fondamentale d'une telle construction commune, le System der Sittenlehre nach den Principien der Wissenschaftslehre, composé deux ans plus tard en 1798, en ceci qu'il touche des questions irréductibles au domaine juridique, offre pour sa part une vue d'ensemble des enjeux d'une telle construction commune. Dans cet ouvrage, suite à la déduction du principe de la moralité (Ch. I) et à celle de l'applicabilité d'un tel principe (Ch. II), Fichte aborde finalement ce qu'il appelle "léthique au sens strict [Sittenlehre im engern Sinne] $»^{40}$ (Ch. III), c'est-à-dire l'application effective et systématique du principe de la moralité; ce qui nous intéresse ici, c'est la deuxième section de ce chapitre qui porte « sur l'élément matériel de la loi morale », puisque c'est là où Fichte va identifier les structures qui permettent une véritable concrétisation de l'autonomie et donc de l'intersubjectivité - dès lors que, comme l'avançait déjà la GNR, on ne peut se poser comme libre (comme autonome) que dans un rapport intersubjectif. ${ }^{41}$

Si l'intersubjectivité se pose comme un problème en fonction de l'action réciproque originaire reprise dans la Sittenlehre sous la dénomination de «limitation originaire ${ }^{42}$, sa mise en œuvre effective exige la formation d'une communauté (l'intersubjectivité concrète) ; du point de vue de l'éthique, cela veut dire que l'autonomie de la raison dépend de cet engagement mutuel puisque « vivre et demeurer en société $»^{43}$ est une condition sine qua non de la réalisation de l'autonomie de la raison. Fichte identifie trois dimensions dont l'articulation permet la concrétisation d'un espace commun : la religion (dimension symbolique) ; le droit (dimension juridique) ; et le forum des savants (dimension de la production culturelle). ${ }^{44}$

Le premier point fondamental dans l'établissement de cet espace de partage est ce que Fichte appelle le symbole : c'est-à-dire la marque du fait qu' « il y a en général quelque chose de suprasensible et de supérieur à toute nature ». Dans la mesure où « ce sur quoi [les membres d'une collectivité] sont tous d'accord, se nomme son

40 Fichte J. G., Le système de léthique selon les principes de la doctrine de la science, trad. par P. Naulin, Paris, PUF, 1986, p. 151 (GA, I, 5, p. 147).

41 Cela signifie que si la moralité peut être déduite d'un point de vue formel, sa mise en œuvre effective, léthique, est foncièrement sociale et intersubjective; sur ce caractère fondamentalement concret de l'éthique fichtéenne, voir l'étude classique de Georg Gurvitsch, Fichtes System der konkreten Ethik, Hildesheim / Zürich / New York, Georg Olms Verlag, 1984.

42 Fichte J. G., Le système de l'éthique selon les principes de la doctrine de la science, op. cit., p. 215 (GA, I, 5, p. 205).

43 Ibid., p. 224 (GA, I, 5, p. 212).

44 Le développement complet de l'argument figure dans : Fichte J. G., Le système de léthique selon les principes de la doctrine de la science, op. cit., pp. 225-241 (GA, I, 5, pp. 213-227). 
symbole", le partage d'un bassin symbolique est ce qui permet à une collectivité de se rassembler autour d'une fin commune. Cette finalité commune dépassant les individualités et constituant leur point d'articulation met en relief ce qui est sous-jacent à la formation d'une communauté en montrant de la sorte, d'une part, que toute communauté s'instaure et se maintient à l'intérieur d'un espace symbolique qui donne un horizon d'action à ses membres et, d'autre part, que ce symbole fonctionne comme une sorte de boussole orientant les forces dans la construction de l'espace commun. Le contenu de ce symbole change évidemment en fonction du contexte sociohistorique, c'est pourquoi Fichte insiste sur le fait qu'il doit pouvoir se transformer continuellement. Ce qui pourtant ne peut pas changer, c'est la présence même d'un symbole quelconque, puisqu' «à défaut d'un accord sur une chose quelconque, aucune action réciproque en vue de produire des convictions communes ne serait possible $»^{45}$.

Si le symbole donne un horizon de partage possible, il ne fournit aucune garantie d'une véritable limitation réciproque assurant la coexistence au sein de cet espace de partage. C'est ici qu'intervient la dimension juridique dont l'opérateur central est ce que Fichte, en s'inspirant de la tradition rousseauiste, appelle le « contrat social ", c'est-à-dire " la convention indiquant comment les hommes doivent pouvoir s'influencer mutuellement ${ }^{46}$; c'est à travers ce contrat que la coexistence des forces est assurée et continuellement protégée, puisque ce contrat assure la soutenabilité de l'espace communautaire en tant que tel. ${ }^{47}$

A part le symbole et le contrat, Fichte souligne encore l'importance d'un troisième élément crucial à la construction de l'espace communautaire, à savoir : l'institution d'un forum au sein duquel « tout le possible doit être pensé et examiné avec une liberté absolue et sans limites ", un lieu où il ne faut se soumettre à aucune autorité et où il n'y a " pas d'autre juge que le temps et le progrès de la culture $»^{48}$. Ce forum est ce que Fichte appelle la République des Savants, qui trouve une forme institutionnelle concrète dans l'université ; laquelle joue ainsi un rôle fondamental au sein de cet espace communautaire.

Si le premier moment de la reconnaissance introduit la saisie du problème de la construction d'un espace communautaire, le second moment articule les trois dimen-

\footnotetext{
Ibid., pp. 231-232 (GA, I, 5, pp. 218-219).

Ibid., p. 227 (GA, I, 5, p. 215).

47 C'est pourquoi, selon la belle formulation d'un interprète, « le contrat social, tel que Fichte le conçoit, implique, au-delà de la simple association, une solidarité effective et active que les citoyens exercent les uns envers les autres sous légide de l'État essentiellement protecteur. ", cf. Fischbach Franck, Fondement du droit Naturel - Fichte, Paris, Ellipses, 2000, p. 39.

48 Fichte J. G., Le système de l'éthique selon les principes de la doctrine de la science, op. cit., pp. 236-239 (GA, I, 5, pp. 223-225).
} 
sions rendant possible la construction effective d'un tel espace : (i) un horizon symbolique commun; (ii) une garantie de protection mutuelle; (iii) un forum de production du savoir. Les deux moments de la reconnaissance sarticulent de la façon suivante :

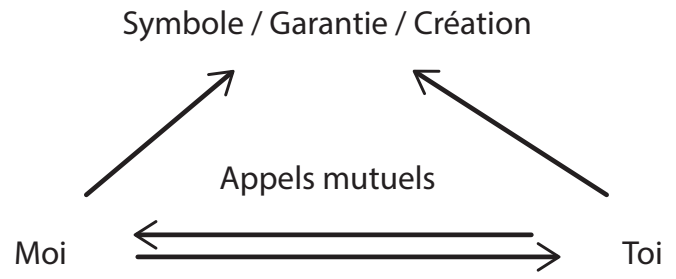

Ainsi, à la différence du premier moment de la reconnaissance où le problème de l'espace commun était posé par le sentiment d'une résistance mutuelle entre les puissances humaines, maintenant, l'on dispose d'outils conceptuels (symbole, normes et créativité) pour construire une solution au problème posé. L'articulation entre le normatif et le socio-ontologique se joue ici au carrefour entre le réseau d'appels exigeant l'autolimitation (d'où la teneur normative du premier moment l'impératif d'autolimitation) et l'horizon de l'engagement effectif dans la construction d'un espace de partage (où s'opère une construction d'un espace socio-ontologique commun - la praxis politique); toujours est-il que tous les deux restent irréductibles l'un à l'autre puisque l'appel ne pose que le problème dont la solution exige l'engagement dans une praxis collective.

\section{L'apport d'une telle approche}

Nous avons vu dans la session précédente que la reconnaissance chez Fichte contient (a) un premier moment originaire où il est question de saisir le problème de la construction d'un espace de partage et (b) un deuxième moment de lélaboration effective d'un tel espace au sein de ses dimensions symbolique, juridique et créatrice. Cette première distinction crée un espace d'écart entre, d'un côté, le problème posé par l'appel d'autrui et, de l'autre, la mise en œuvre effective de dispositifs de reconnaissance visant à affronter le problème : cela ouvre une possibilité d'articulation entre le normatif, le devoir d'entrer en communauté pour réaliser concrètement l'autonomie, et l'ontologique, ce qui est en jeu sur la scène concrète de la construction effective d'un espace communautaire.

Si l'on reprend maintenant l'idée d'après laquelle les théories contemporaines de la reconnaissance sont incapables de rendre compte de l'horizon constitutive- 
ment conflictuel de toute demande de reconnaissance, soit (i) en fonction d'un défaut ontologique, issu d'une insistance exagérée sur le normatif empêchant la problématisation de la reconnaissances de sujets en tant que sujets, soit (ii) en raison d'un défaut normatif, issu d'une exagération du moment socio-ontologique rendant l'écart critique peu compréhensible ; alors, on peut voir comment la théorie fichtéenne fait droit à la conflictualité inhérente aux structures de reconnaissance. En effet, l'irréductibilité entre le niveau où le problème se pose et celui où l'on mobilise des outils pour y répondre indique que la conflictualité se constitue au moment de construire des réponses (religieuses, juridiques et culturelles) à l'exigence de reconnaissance issue de la présence d'autrui; en d'autres termes, ce qu'il y a de normatif dans l'appel issu de la présence humaine devant moi est irréductible au domaine socio-ontologique constitué, tandis que le caractère foncièrement ontologique de la scène sur laquelle la reconnaissance se joue est, pour sa part, irréductible à l'appel.

Par conséquent, l'irréductibilité des deux moments permet, d'une part, (i') d'affronter le défaut ontologique, puisque le moment normatif issu de l'impératif d'autolimitation n'empêche pas de thématiser le problème de la reconnaissance des sujets en tant que sujets; en effet, la simple présence humaine pose déjà le problème de la construction d'un espace rendant possible la transformation des humains présents en des consciences reconnues. D’autre part, une telle irréductibilité permet également, (ii') d'affronter le défaut normatif, dès lors que l'espace socio-ontologique constitué n’empêche pas l'écart critique, en ceci que l'appel normatif de la reconnaissance est posé par la simple présence d'autrui; l'écart critique est possible, même au sein d'un espace d'omniprésence de l'idéologie, par l'appel issu de la présence humaine.

Au demeurant, la richesse d'un tel modèle nous permet d'aller encore plus loin. En effet, dans la mesure où le modèle fichtéen identifie le problème de la concrétisation de la reconnaissance à celui de la construction d'un espace communautaire, il nous permet de resituer la question de la reconnaissance à l'intérieur d'un paysage plus large qui inclut non seulement le domaine du droit et du politique mais aussi celui de la dimension symbolique et de la production du savoir - deux dimensions fondamentales dans la constitution et le maintien d'une communauté. Un tel élargissement du paysage d'articulation de la question nous permet d'inclure dans les discussions concernant la reconnaissance non seulement le domaine de la loi et du pouvoir politique, mais aussi ce que l'on pourrait appeler en un sens large le domaine de la culture - ce qui inclut la religion et les institutions d'apprentissage.

Cela ouvre les portes à une problématisation non seulement de la conflictualité inhérente à la formation de structures de réponse aux appels de reconnaissance, 
mais aussi à celle d'une incompréhension systématique qui peut ressortir de telles structures en fonction de la négligence des rapports entre les dimensions symbolico-culturelle et juridico-politique. En effet, si la construction d'un espace communautaire mobilise tout un réseau symbolique contenant une certaine conception de la finalité commune et si ce n'est quau sein d'un tel réseau que les sujets agissants prennent conscience de leurs individualités, alors la reconnaissance ne se joue pas seulement au niveau de l'acceptation d'une identité, mais aussi à celui du processus de constitution des identités ; autrement dit, l'exigence issue de la présence d'autrui n'est pas celle de reconnaître l'identité constituée d'un être libre, mais celle de reconnaître son pouvoir de participer lui-même à un processus de constitution identitaire.

En se focalisant sur le processus de constitution identitaire au sein de la communauté, l'approche fichtéenne permet notamment d'explorer les idées du suprasensible qui sont opératoires au sein d'un tel déploiement (la dimension symbolique) ainsi que le rôle fondamental des intuitions de production du savoir (la dimension créatrice) au sein desquelles les réseaux conceptuels de l'intersubjectivité peuvent être remis en question et critiqués. Faute d'une possibilité de remise en question de tels réseaux, les structures de reconnaissance risquent de reproduire de façon inerte des incompréhensions du rapport intersubjectif - une déviation qui, sur la longue durée, ne tend pas à l'auto-guérison comme l'Esprit chez Hegel, mais plutôt à l'inertie d'un blocage.

C’est par ailleurs précisément cette inertie que Fichte appelle « le mal radical », en ceci qu'il s'agit d'une force poussant toute nature à « demeurer ce qu'elle est » face à n’importe quelle activité lui étant opposée, elle constitue même le trait fondamental de tout ce qui tend à rester en repos et donc de tout être. Cette tendance est ce qui pousse lêtre humain et ses structures de rapports intersubjectifs à des automatismes incompatibles avec une vraie vie. ${ }^{49}$ Elle se manifeste, selon le philosophe, sous la forme d'une «lâcheté » : (a) corporelle, qui est à l'origine de la peur devant "l'effort corporel de la résistance »; et (b) intellectuelle, conduisant à « la soumission des propres idées à l’autorité ». Un corps incapable de résister et un intellect incapable de penser ne peuvent quempêcher l'expression de toute force vitale ; lêtre humain est dès lors pris en otage par un automatisme inerte.

49 Les automatismes seraient ainsi ce qui empêche « la rationalité immanente ", dont parlait Hegel, de s'exprimer : une telle rationalité est certes là de façon inconsciente, puisque, comme l'a bien fait remarquer un interprète "le Moi Absolu précédant toute conscience est le nom fichtéen de l'inconscient» (Cf. Vaysse J.-M., L'inconscient des Modernes - Essai sur l'origine métaphysique de la psychanalyse, Paris, Gallimard, 1999, p. 237) ; cependant elle est exposée au risque constant d'avoir son mouvement bloqué à cause d'une tendance interne à l'inertie. 
Un tel automatisme conduit, d'après Fichte, à la production d'un réseau de fausses croyances face à une oppression jamais directement affrontée. ${ }^{50}$

On peut ainsi dire que, de façon analogue au modèle hégélien, la reconnaissance n'advient ici que si l'on affronte directement ce qui nous opprime, mais, contrairement à un tel modèle, on attire l'attention sur cette tendance chez lêtre humain à la reproduction systématique du même, se manifestant aussi bien au niveau matériel qu'au niveau intellectuel et s'exprimant potentiellement dans un réseau de fausses croyances qui joue un rôle non-négligeable dans l'oblitération de l'oppression. On entrevoit une sorte de "ruse de la raison » à l'inverse, c'est-à-dire une tendance non pas à l'auto-guérison de l'Esprit, mais plutôt à la reproduction systématique de la blessure faisant courir le risque de la contamination du réseau de croyances au sein duquel le mouvement de l'Esprit se déploie. ${ }^{51}$

Si les réseaux conceptuels tendent, en effet, à se sédimenter ontologiquement, ce qui peut conduire à fausser systématiquement notre appréhension des rapports intersubjectifs, alors il est essentiel que l'on dispose, au sein de l'espace communautaire, d'institutions qui nous permettent de remettre en question les concepts à partir desquels l'espace commun s'organise, d'où l'importance centrale d'inclure dans la problématique de la reconnaissance la dimension incarnée par les forums de discussions savantes. ${ }^{52}$ Par conséquent, si la théorie hégélienne présente l’avan-

50 Fichte J. G., Le système de l'éthique selon les principes de la doctrine de la science, op. cit., pp. 192-195 (GA, I, 5, p. 183-186).

51 Ce point indique un contraste substantiel entre le projet philosophique fichtéen et celui hégélien, se manifestant dans leurs visions de la tâche de la philosophie. D’un côté, nous avons la célèbre image de la chouette de Minerve qui ne prend son envol qu’au crépuscule afin de reconstruire le processus de formation de l'Esprit du point de vue d'un mouvement accompli (Cf. Hegel G. W. F., Principes de la Philosophie du droit, p. 41) ; d'un autre côté, l'idée que les grands philosophes puissent « briser les plus fortes chaînes de l'humanité » (Cf. Fichte J. G., Considérations sur la Révolution Française, trad. par J. Barni, Paris, Payot, 1974, p. 127 - GA, I, 1, p. 255) puisque la tâche d'une vraie philosophie est celle d'opérer une véritable "transformation de nous-mêmes [eine Umschaffung unser selbst] » (Cf. Fichte J. G., La Théorie de la Science. Exposé de 1804, trad. par D. Julia, Paris, Aubier, 1993, p. 31 - GA, II, 8, p. 18). D'un côté, une philosophie consacrée à reconstruire un processus tel qu'il s'est déroulé jusqu’à présent ; d'un autre côté, une philosophie dédiée à transformer l'avenir de l'esprit : les deux compréhensions ne sont certes pas incompatibles (on pourrait dire que la reconstruction du passé est précisément ce qui ouvre la porte à un nouvel avenir), cependant l'on continue à mettre l'accent, d'une part, sur les outils conceptuels de l'explication et, d'autre part, sur les outils conceptuels de la libération.

52 En ce sens, la philosophie fichtéenne contient un puissant pouvoir transformateur, puisqu'en déconstruisant les structures conceptuelles bloquant le libre mouvement de l'intuition, elle engendre en même temps l'ouverture de l'horizon de l'expérience à d'autres modes de perception; ce qui a conduit un interprète à ranger la philosophie fichtéenne à côté de celle du maitre spirituel de l'hindouisme, Adi Sankara, en tant que véritable philosophie transformatrice, car elle " does not represent a view of reality, but a view which transform reality " (cf. Taber John A., Transformative Philosophy. A Study of Sankara, Fichte, and Heidegger, Honolulu, University of Hawaii Press, 1983, 
tage de nous faire voir la relation constitutive entre conflit et reconnaissance, la théorie fichtéenne nous permet encore d'explorer les réseaux symbolico-culturels au sein desquels les structures de reconnaissance sont mises en marche afin de cerner la possibilité, toujours réelle, d'une falsification systématique de nos façons de concevoir la conflictualité.

Si l'on veut problématiser les rapports intersubjectifs dans un monde peuplé par des univers symboliques aussi divers que le nôtre, il convient d'inclure dans les discussions portant sur la reconnaissance le rôle de la dimension symbolico-culturelle et des institutions de production du savoir dans le processus de constitution identitaire ; pour ce faire, la philosophie fichtéenne se révèle une puissante alliée.

Luis Fellipe Garcia est Docteur en Philosophie (Université catholique de Louvain, UCLouvain) et travaille sur la philosophie classique allemande et sur la pensée décoloniale. Il a publié notamment La philosophie comme Wissenschaftslehre (Georg Olms coll. Europaea Memoria, 2018) et plusieurs articles dans des revues scientifiques et dans des ouvrages collectifs.

E-mail : luisfellipegarcia@gmail.com

p. 102). Cette capacité à transformer la réalité fait de la pensée fichtéenne une source féconde de dispositifs critiques pour problématiser les structures cognitives obnubilant la perception mutuelle des membres d'univers symboliques distincts ; la philosophie fichtéenne se révèle ainsi comme une importante alliée d'un projet de décolonisation de la pensée en ceci quelle « opens our performances to different languages, different ways of speaking, and different kinds of vision, ones completely other to our own " (cf. Goddard Jean-Christophe, "The Beech and the Palm Tree - Fichte's Wissenschaftslehre as a Project of Decolonization ", in J. Carew et S. Macgrath (Org.), Rethinking German Idealisme, London, Macmillan, 2016, p. 161). 